\title{
Formation and destruction of polycyclic aromatic hydrocarbon clusters in the interstellar medium
}

\author{
M. Rapacioli ${ }^{1}$, F. Calvo ${ }^{2}$, C. Joblin ${ }^{1}$, P. Parneix ${ }^{3}$, D. Toublanc ${ }^{1}$, and F. Spiegelman ${ }^{2}$ \\ ${ }^{1}$ Centre d'Étude Spatiale des Rayonnements, CNRS-Université Paul Sabatier, Observatoire Midi-Pyrénées, 9 Av. du colonel Roche, \\ BP 4346, 31028 Toulouse Cedex 4, France \\ e-mail: mathias.rapacioli@chemie.tu-dresden.de \\ 2 Laboratoire de Chimie et Physique Quantiques, IRSAMC, Université Paul Sabatier, 118 Route de Narbonne, 31062 Toulouse \\ Cedex, France \\ ${ }^{3}$ Laboratoire de Photophysique Moléculaire, CNRS Bât. 210, Université Paris-Sud, 91405 Orsay Cedex, France
}

Received 11 April 2006 / Accepted 20 June 2006

\section{ABSTRACT}

\begin{abstract}
Aims. The competition between the formation and destruction of coronene clusters under interstellar conditions is investigated theoretically.

Methods. The unimolecular nucleation of neutral clusters is simulated with an atomic model combining an explicit classical force field and a quantum tight-binding approach. Evaporation rates are calculated in the framework of the phase space theory and are inserted in an infrared emission model and compared with the growth rate constants.

Results. It is found that, in interstellar conditions, most collisions lead to cluster growth. The time evolution of small clusters (containing up to 312 carbon atoms) was specifically investigated under the physical conditions of the northern photodissociation region of NGC 7023. These clusters are found to be thermally photoevaporated much faster than they are reformed, thus providing an interpretation for the lowest limit of the interstellar cluster size distribution inferred from observations. The effects of ionizing the clusters and density heterogeneities are also considered. Based on our results, the possibility that PAH clusters could be formed in PDRs is critically discussed.
\end{abstract}

Key words. astrochemistry - molecular processes - ISM: molecules - reflection nebulae - dust, extinction

\section{Introduction}

Many astronomical objects show a distinct set of emission bands in the mid-infrared range, known collectively as the unidentified infrared emission features. The most intense of these features, the Aromatic Infrared Bands (AIBs) falling at 3.3, 6.2, "7.7", 8.6, 11.3 and $12.7 \mu \mathrm{m}$, are the signatures of aromatic $\mathrm{CC}$ and $\mathrm{CH}$ bonds. They are observed systematically from different regions of the interstellar medium (ISM) irradiated by UV photons. The carriers of these bands have been identified as Polycyclic Aromatic Hydrocarbons (PAHs) some twenty years ago by Léger \& Puget (1984) and Allamandola et al. (1985).

Boulanger et al. (1990) and Bernard et al. (1993) analysed the emission in the IRAS photometric bands measured for several molecular clouds, and suggested that free-flying PAHs are produced by photoevaporation of larger grains. The observation of a mid-infrared continuum in the reflection nebula Ced 201 has been attributed by Cesarsky et al. (2000) to Very Small carbonaceous Grains (VSGs) producing the AIB carriers. More recently, in their study of the two reflection nebulae NGC 7023 and $\rho$ Ophiucus-SR3, Rapacioli et al. (2005b) showed that PAH molecules are produced by the destruction of small carbonaceous grains inside molecular clouds, these grains being interpreted as PAH clusters. A minimal size of 400 carbon atoms per cluster was inferred from the analysis of astronomical observations (Rapacioli et al. 2005b). A plausible scenario is that these grains undergo photo-evaporation at the edge of clouds, thus leading to isolated PAHs. These observations motivate theoretical and experimental studies on the physical properties of PAH clusters, such as structure, stability or aggregation.

Small clusters of PAH molecules have been studied by several authors in the past, both experimentally (Benharash et al. 1999; Song et al. 2003; Piuzzi et al. 2002; Miller et al. 1984) and theoretically (van de Waal 1983; Gonzales \& Lim 1999; Marzec 2000; Piuzzi et al. 2002; Grimme 2004). Most of these studies have focused on rather small species, usually containing no more than a few tens of carbon atoms, far below the limit of 400 carbon atoms per VSG as suggested by Rapacioli et al. (2005b). Studies on larger systems have been initiated only lately, motivated by the astrophysical context. Clusters of coronene molecules containing up to 20 units have been produced in a gas aggregation source (Bréchignac et al. 2005; Schmidt et al. 2006). Interestingly, the authors showed that the dissociation of coronene clusters induced by multiphoton excitation of a near-UV laser proceeds via the ejection of van der Waals-bonded coronene units.

In a previous work (Rapacioli et al. 2005a), we investigated the stable structures of PAH clusters containing up to 800 carbon atoms (32 coronene molecules). The considered PAHs ranged from pyrene $\left(\mathrm{C}_{16} \mathrm{H}_{10}\right)$ to circumcoronene $\left(\mathrm{C}_{54} \mathrm{H}_{18}\right)$. The most stable cluster structures generally result from stacking the $\mathrm{PAH}$ molecules, first in a one-dimensional pattern at small cluster sizes. Above some critical size (depending on the PAH itself), structures more stable than the single stack are found, consisting of shorter stacks lying next to each other, and growing as two-, eventually three-dimensional structures. In large PAH clusters, 
the multiple ways of arranging the short stacks lead to a competition between stable structures.

Our present interest lies in the formation and destruction of PAH clusters in the ISM, and particularly the competition between these two processes. The coronene molecule $\left(\mathrm{C}_{24} \mathrm{H}_{12}\right)$ provides a convenient example of large PAHs, especially from the computational point of view, and is used in the present work as a prototype for interstellar PAHs. We wish to address here the following questions:

(i) what are the astrophysical conditions that favour the nucleation of PAH clusters?

(ii) how much energy is required for thermal evaporation of these clusters?

(iii) how do the rates of formation and destruction compare for these clusters under interstellar conditions?

In this paper, we focus on small neutral coronene clusters containing up to 312 carbon atoms, or 13 molecules. In particular, we attempt to offer a physical explanation for the existence of the observed lower limit to the interstellar PAH cluster size distribution of 400 carbon atoms per cluster (Rapacioli et al. 2005b).

Addressing these questions requires a variety of theoretical methods to be used. Nucleation processes are explicitly simulated with an extended version of the tight-binding (TB) model initially developed by Van Oanh et al. (2002) for single PAHs. Concerning the thermal stability of coronene clusters, the rates of unimolecular evaporation as well as the photoemission rates in the infrared are obtained and compared. The evaporation rates are obtained in the statistical framework of the phase space theory (Chesnavich \& Bowers 1977). The competition between evaporation and IR emission is calculated using a master equation modeling based on microcanonical statistics (Joblin et al. 2002). In the light of these calculations, we discuss several chemical scenarios to interpret the observations of the reflection nebulae (Rapacioli et al. 2005b).

The paper is organized as follows. In the next section, we describe the atomic model and discuss the numerical simulation of coronene clusters growth by unimolecular nucleation. In Sect. 3, the evaporation rates of coronene clusters are calculated. In Sect. 4, we study the competition between evaporation and IR emission. In Sect. 5, the photophysics of a coronene cluster in a radiation field is simulated. In Sect. 6, we study the competition between formation and destruction of coronene clusters under interstellar conditions. We also highlight the astrophysical implications of our results for possible models of chemical evolution in the photodissociation regions. In particular, the possible roles of ionization and density heterogeneities in the PDR in the existence and stability of PAH clusters are discussed. Some conluding remarks are given in Sect. 7.

\section{Cluster formation process}

In this section, we consider the formation of neutral coronene clusters by collision of a single molecule with a smaller cluster or with another single molecule. In the ISM, collisions occur in a very rarefied gas and the time lapse between successive UV absorption events is in general much longer than the IR emission timescale. Therefore the PAH molecules and their clusters have enough time to cool down through IR emission, and can be considered as initially cold in their ground electronic and vibrational states. This energy will be transferred to intermolecular modes, then to the intramolecular modes. Intramolecular vibrational energy redistribution (IVR) is likely to be the major source for dissipating the initial energy, having thus strong consequences on the collision products. Hence it is important to take this redistribution into account in molecular simulations.

In this paper, we will consider that a new cluster is formed upon collision if it has time to reach thermal equilibrium before further dissociating. The relaxation of a thermalised cluster heated by collision or by photoabsorption is discussed in Sect. 3 . We describe hereafter the atomic model that was implemented for the present problem.

\subsection{Atomic model}

Describing the transfer of the collisional energy toward intermolecular and intramolecular modes requires both types of degrees of freedom to be correctly accounted for. Clusters of coronene molecules are modeled using the combination of an explicit classical force field to describe the intermolecular interactions, along with a quantum tight-binding model for the intramolecular interactions within each molecule. The total Hamiltonian for a system with $N_{\text {mol }}$ PAH molecules is written:

$H=\sum_{i=1}^{N_{\mathrm{mol}}} H_{\mathrm{intra}}^{i}+H_{\mathrm{inter}}$

In this equation $H_{\text {intra }}^{i}$ is the intramolecular Hamiltonian of molecule $i$, which only explicitly depends on the positions of the atoms in this molecule. We use for $H_{\text {intra }}^{i}$ the tight-binding model developed by Van Oanh et al. (2002). This simple quantum model was parameterized to reproduce energetic and vibrational properties of isolated PAH molecules. We did not change its original parameters.

$H_{\text {inter }}$ is the intermolecular potential resulting from the repulsion-dispersion forces as well as the electrostatic interactions between partial charges on the atoms of different molecules. Following our previous work (Rapacioli et al. 2005a), we use a simple Lennard-Jones (LJ) + point charges potential, the charges being determined to reproduce Density Functional Theory (DFT) calculations on an isolated molecule. The LJ parameters are taken from van de Waal (1983). Further details about the intermolecular potential, including a discussion about the sensitivity of the results with respect to the potential parameters, can be found in Rapacioli et al. (2005a).

\subsection{Simulation details}

The classical equations of motion on the Born-Oppenheimer potential energy surface have been numerically integrated using the fifth-order Adams-Moulton predictor-corrector algorithm. The time step was chosen between 0.05 fs and 0.15 fs depending on the collision energy. The computational effort required to diagonalize the multiple TB Hamiltonians was distributed among many processors on parallel computers in the OpenMP framework. As stated previously, the reactants are assumed to be cold, their vibrational energies being taken as less than $80 \mathrm{~K}$ (much lower than the collisional energy). The physical parameters, which characterize a collision in the centre of mass reference frame, are the collisional energy (translational plus rotational components), the impact parameter, the angular momentum of each fragment and their initial orientations. The statistical information obtained from the simulations was limited due to the still relatively heavy numerical cost involved in computing the TB energies and forces. Therefore we restricted our study to 
the following representative situations, assuming this restriction has a minor impact on the collision products:

- all collisions are at zero impact parameter (no orbital angular momentum);

- the total angular momentum of the system (incident molecule+cluster) is zero;

- the collisional energy is equally distributed between the translational energy and the rotational energy of the reactants.

At a given collisional energy, the free mechanical parameters are thus reduced to the initial orientations of the molecule and the cluster and the direction of the angular momentum of the cluster. We did not attempt to determine quantitative cross sections for the collision processes.

About 30 trajectories have been performed for each collisional energy in order to sample these parameters and to gather statistics on the outcome of the collisions. For simplicity the collision of reactants with sizes $p$ and $p^{\prime}$, respectively, leading to a set of fragments with sizes $d, d^{\prime}, d^{\prime \prime} \ldots$ will be referred to as $p+p^{\prime} \rightarrow d+d^{\prime}+d^{\prime \prime}+\cdots$, where the parents and products are ordered by increasing size.

In practice the simulation ends when either one of the two following situations happens:

- after the collision, fragmentation occurs and one or more molecules are ejected far from the others;

- the instantaneous intra- and intermolecular temperatures cross each other, meaning that the product cluster has absorbed the collisional energy and has reached thermal equilibrium.

Other situations have not been observed.

\subsection{Results}

Figure 1 shows the nucleation probability of simulated collisions between two coronene molecules as a function of collisional energy, that is the chance that a collision leads to the formation of a dimer as $1+1 \rightarrow 2$. The probability that the collision fails in producing a dimer $(1+1 \rightarrow 1+1)$, also plotted in Fig. 1, complements the nucleation probability.

These results show that the probability of forming a dimer decreases with increasing collisional energy. This is not surprising, since collisions at higher energy tend to make the two molecules bounce of each other. Nucleation is efficient, especially at low energies. For instance, the probability of forming a coronene dimer exceeds $50 \%$ when the collisional energy is lower than $3.6 \mathrm{eV}$. This rather high energy threshold corresponds to the average collisional energy of a gas at a temperature of $13500 \mathrm{~K}$.

The probabilities of successfully forming a larger cluster or of destroying the initial cluster from a collision between a single coronene and a stack of 2 or 3 molecules are shown in Figs. 2 and 3 , respectively. For these clusters, the probability of a neutral event, that is when the collision products are identical to the parents in terms of their size, is also plotted. The multifragmentation of the collision product into single molecules only was not observed in the case of the tetramer.

As in the case of dimer formation, the probability of growing the initial cluster decreases monotonically with increasing collisional energy. A monotonic increase is also observed for the probability of ending with smaller clusters. This allows us to define a critical energy for which these two probabilities are the

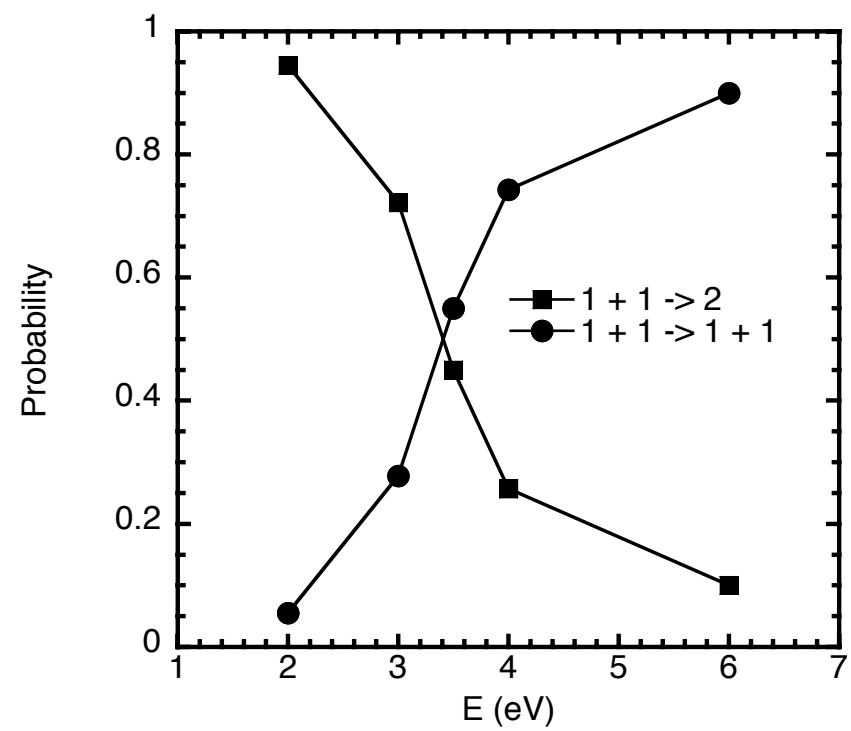

Fig. 1. Probabilities $p_{+}^{0}$ and $p_{n}^{0}$ that a collision between two coronene molecules forms a dimer $(1+1 \rightarrow 2)$ or not $(1+1 \rightarrow 1+1)$, as a function of the collisional energy. Each data point is averaged over 30 simulations. Lines are drawn to guide the eye.

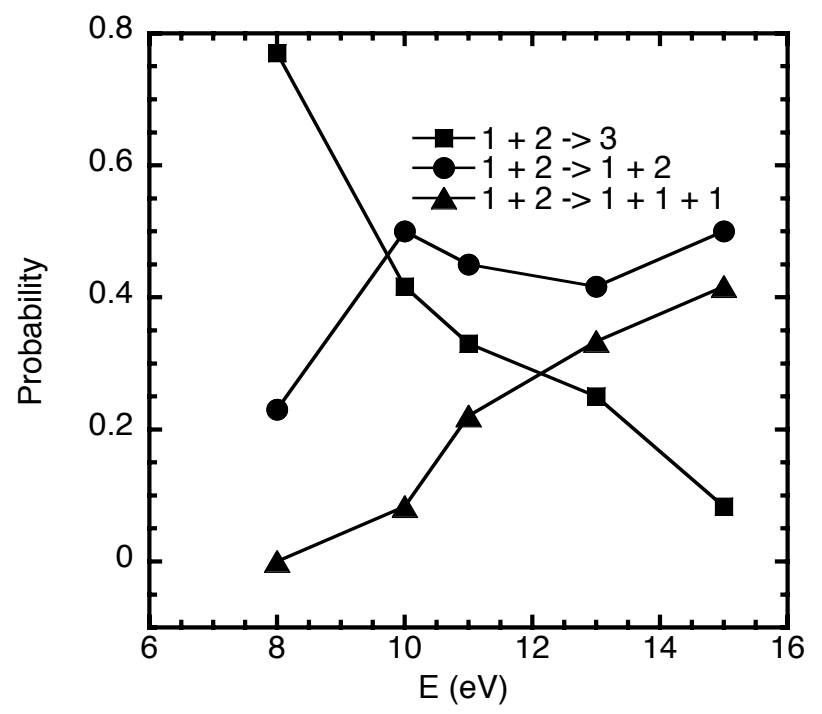

Fig. 2. Probabilities $p_{+}^{0}, p_{n}^{0}$, and $p_{-}^{0}$ that a collision of a single coronene on a dimer forms a trimer $(1+2 \rightarrow 3)$, leaves a dimer $(1+2 \rightarrow 1+2)$, or fragments into 3 molecules $(1+2 \rightarrow 1+1+1)$, as a function of the collisional energy. Each data point is averaged over 30 simulations. Lines are drawn to guide the eye.

same. This energy approximately equals $12.4 \mathrm{eV}$ for the $1+2$ reaction and $14.2 \mathrm{eV}$ for $1+3$. The increase of the critical energy with cluster size is expected since a larger number of modes are available to convert the collisional energy. In our simulations, the range of collisional energies was not chosen on the basis of astrophysical values but only to allow enough statistics to be gathered in the calculations. Even though the mean collisional energies encoutered in the interstellar medium are usually much lower (typically in the $0.01-0.1 \mathrm{eV}$ range), we extrapolate below the values corresponding to the smallest calculated collisional energies to collisions with lower energies. This approximation leads to some underestimation of the cluster growth, which we estimate to be no more than $25 \%$ since the sticking probability is at least 0.75 (as seen from Figs. 1-3). 


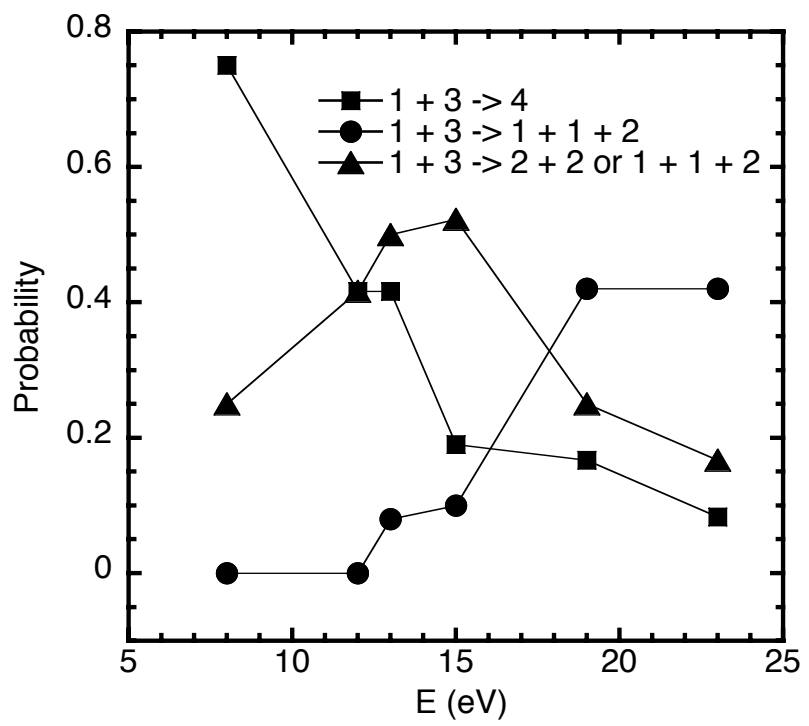

Fig. 3. Probabilities $p_{+}^{0}, p_{n}^{0}$, and $p_{-}^{0}$ that a collision of a single coronene on a trimer forms a tetramer $(1+3 \rightarrow 4)$, leaves a trimer $(1+3 \rightarrow 1+3)$, destroys the inital cluster $(1+3 \rightarrow 2+2$ or $1+3 \rightarrow 1+1+2)$, as a function of the collisional energy. Each data point is averaged over 30 simulations. Lines are drawn to guide the eye.

\section{Evaporation of a neutral isolated cluster}

A thermalised isolated coronene cluster with an excitation energy $E_{\text {tot }}$ can release a part of this excess energy via dissociation, evaporation and IR emission. We will assume that the cluster remains on the ground state electronic potential surface. In this section, we study the evaporation mechanism, which in turn requires an estimation of the characteristic time for the energy redistribution between inter- and intramolecular modes. In the next section, we study the competition between the different relaxation mechanisms involving molecular decay or infrared emission.

\subsection{Vibrational redistribution}

Neglecting the influence of the excited states, collisions and UVvisible photon absorption under astrophysical conditions leads to an increase of the internal energy of the cluster, which can then be dissipated either by evaporation and/or IR emission. The energy redistribution between inter- and intramolecular vibrational modes is required for the cluster to be considered in thermal equilibrium upon nucleation of a molecule. Similarly, the absorption of a photon by a single molecule in the cluster will tend to heat the intramolecular modes of this specific molecule first by internal conversion, before the excess energy flows into intermolecular modes, and subsequently into the intramolecular modes of the other molecules. The time scales involved in these fundamental redistribution processes should be considered as reference data in the next stages of our investigation.

As a first application of our model for PAH clusters, we have simulated the energy redistribution of intramolecular energy toward intermolecular modes by heating a single molecule in the cluster and monitoring the amount of kinetic energy in all other molecules as a function of time. The kinetic energy provides a direct estimate of the vibrational or thermal energy. We consider redistribution in the (coronene) $)_{5}$ cluster as a typical example. This cluster consists of a short stack, for which either the outermost or the central molecule are chosen to carry the initial thermal excitation. The starting configuration of the cluster is

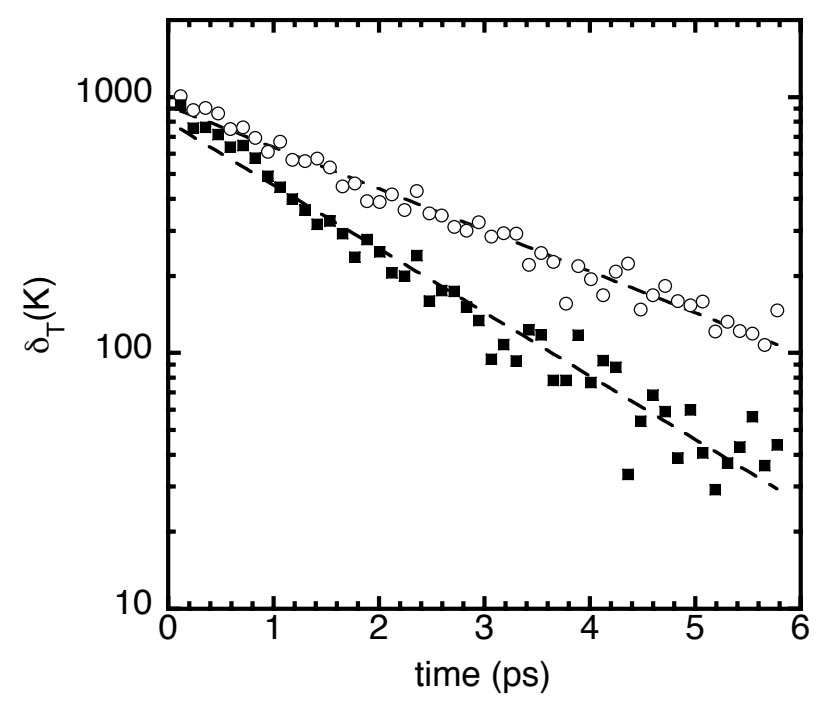

Fig. 4. Root mean squares $\delta_{T}$ of the kinetic energies of the molecules in a cluster of five molecules. At $t=0$, the initially kinetic energy excess $(13 \mathrm{eV})$ is localized in the outermost (circles) or central (squares) molecule within the stack. The dashed lines are linear fits.

taken as the lowest-energy structure, and no extra kinetic energy is given to the intermolecular modes. To quantify the redistribution of energy into vibrational modes of all molecules in the cluster, we introduce the root mean square fluctuation $\delta_{T}$ of the kinetic temperatures of the molecules in the cluster:

$\delta_{T}(t)=\frac{1}{N_{\mathrm{mol}}} \sqrt{\sum_{i=1}^{N_{\mathrm{mol}}}\left(T_{i}(t)-\langle T(t)\rangle\right)^{2}}$

where $T_{i}(t)$ is the average kinetic temperature restricted to molecule $i$ and $\langle T(t)\rangle$ is the usual average kinetic temperature of the entire system. $\delta_{T}(t)$ provides an instantaneous measure of the standard deviation of the individual temperatures of all molecules in the cluster. Initially this quantity is large, since only one molecule carries kinetic energy. Eventually the cluster reaches equilibrium and the temperatures of all molecules in the cluster are identical. The rate at which $\delta_{T}(t)$ decreases yields an estimate for the time scale $\tau$ of energy redistribution in the system, according to $\delta_{T}(t) \sim \delta_{T}(0) \mathrm{e}^{-t / \tau}$.

Figure 4 shows the variations of $\delta_{T}$ as a function of time for $\left(\mathrm{C}_{24} \mathrm{H}_{12}\right)_{5}$ in which the initial excitation is localized on different molecules. The decrease of $\delta_{T}$ is indeed exponential, with decay time constants approximately equal to $\tau=1.47 \pm 0.09 \mathrm{ps}$ when the central molecule is vibrationally excited, and $\tau=2.67 \pm 0.28$ ps when the outermost molecule is excited. These time scales are of the same magnitude as the softest vibrational modes of isolated coronene molecules (Martin 1996). This is not surprising, because the slowest modes constitute a lower limit to the rate at which energy is redistributed homogeneously within all internal degrees of freedom. Finally, the faster redistribution when the central molecule is heated is expected due to the presence of fewer intermolecular modes involved.

\subsection{Evaporation}

Coronene clusters could a priori dissociate into entire molecules, or via intramolecular fragmentation. The loss of a molecule from a coronene cluster (typically $1 \mathrm{eV}$ of binding energy, Rapacioli et al. 2005a) is much more likely to happen than the breaking of chemical bonds, which requires more than $4 \mathrm{eV}$ 
(Joblin et al. 2006). In agreement with the experimental conclusions of Schmidt et al. (2006), we thus only consider here the dissociation corresponding to single molecule evaporation.

Evaporation rates of molecular clusters are not available by direct molecular dynamics simulations except at very high excitation energy. When the extra energy deposited in the cluster is marginally higher than the dissociation energy, fragmentation is a rare event, which can take place over time scales much longer than the typical times that are reachable by conventional molecular simulations. Statistical rate theories are much more convenient for calculating such rates. The phase space theory (PST) developed by Chesnavich \& Bowers (1977) provides an accurate framework for unimolecular dissociation. In previous work, two of us (Calvo \& Parneix 2004) showed that PST predictions were in quantitative agreement with numerical simulations for molecular systems, provided that the rotational and vibrational densities of states (DOS) were correctly evaluated.

\subsubsection{Phase space theory}

The success of PST is essentially due to the rigorous conservation of energy and angular momentum, and to the inclusion of anharmonic vibrational effects. Here we are mainly interested in the determination of absolute rate constants for the evaporation of a single molecule from a thermalized cluster. PST expressions for the rate constant (Jarrold 1991) contain a set of factors that are not always obvious to estimate with accuracy. Instead of assuming specific values for these unknown factors, we follow Weerasinghe \& Amar (1993) and use the results of molecular dynamics simulations at high internal energy to calibrate these unknown factors.

Performing molecular dynamics simulation of the cluster with both inter- and intramolecular interactions is not realistic in the context of thousands of trajectories over time scales governed by the intermolecular motion. Therefore we had to simplify the modeling by freezing intramolecular motion. With this assumption, the molecules are treated as rigid bodies, and larger time steps and algorithmic improvements such as the use of quaternion coordinates allow an efficient simulation of the evaporation process. Even though the rigid body approximation may not be physically relevant at high energies, it calibrates the statistical theory, that we will subsequently use in a lower excitation regime.

In this subsection, energies are thus measured on the intermolecular potential energy surface $H_{\text {inter }}$ only. It is not our goal here to describe the PST formalism in great details, and the reader is refered to the work by Chesnavich \& Bowers (1977), Weerasinghe \& Amar (1993), Calvo \& Parneix (2004) for further information. The differential rate of unimolecular evaporation from a molecular cluster $X_{n+1} \rightarrow X_{n}+X$ (here $X=C_{24} \mathrm{H}_{12}$ ), at total rovibrational energy $E$ and angular momentum $J$, and leading to the loss of kinetic energy $\varepsilon_{\mathrm{tr}}$ into translation $\varepsilon_{\mathrm{t}}$ and rotation $\varepsilon_{\mathrm{r}}$ is expressed as

$R\left(E, J, \varepsilon_{\mathrm{tr}}\right)=C_{0} \frac{\Omega^{\prime}\left(E-E_{0}-\varepsilon_{\mathrm{tr}}\right) \Gamma^{\prime}\left(J, \varepsilon_{\mathrm{tr}}\right)}{\Omega\left(E-E_{\mathrm{r}}\right)}$.

In the above equation $E_{0}$ is the dissociation energy, that is the binding energy difference between the parent and the product clusters, $E_{\mathrm{r}}$ is the parent rotational energy, equal to $B J^{2}$ where $B$ is the rotational constant. $\Omega$ and $\Omega^{\prime}$ are the vibrational densities of states of the parent and product clusters, respectively. $\Gamma^{\prime}$ is the rotational density of states of the two products, obtained from the mechanical constraints on angular momentum at a given translational + rotational energy. An estimation of $\Gamma^{\prime}$ requires treating the products as rigid bodies. $C_{0}$ is a constant that we avoid calculating by performing high energy molecular dynamics simulations for calibration. The rate constant $k(E, J)$ is obtained from the differential rate $R\left(E, J, \varepsilon_{\mathrm{tr}}\right)$ by integration over the available range of $\varepsilon_{\mathrm{tr}}$.

The ingredients of the PST calculations are threefold. First, the vibrational densities of states $\Omega$ and $\Omega^{\prime}$ are calculated using parallel tempering Monte Carlo simulations (Neirotti et al. 2000) analysed with a multiple histogram method. These calculations consist of running independent Monte Carlo trajectories or replicas at different temperatures, occasionnally attempting to swap configurations between adjacent replicas. Here we use 50 different temperatures in the range $10 \mathrm{~K}-1500 \mathrm{~K}$ distributed according to a geometric progression. For each trajectory, $6 \times 10^{5}$ Monte Carlo cycles $(1 \mathrm{MC}$ cycle $=n$ individual steps $)$ are carried out for the calculation, the first $10^{5}$ steps being discarded for equilibration.

The rotational density of states $\Gamma^{\prime}$ requires integrating the number of rotational states $\chi$ with specified internal angular momentum of the products $J_{\mathrm{r}}=J_{1}+J_{2}$ and specified orbital momentum $L$ under the constraint that the translational energy released $\varepsilon_{\mathrm{t}}$ is larger that the centrifugal barrier $\varepsilon^{\dagger}$. The calculation becomes significantly simpler by assuming that the initial angular momentum is small or, more precisely, that the rotational energy is small with respect to the vibrational energy. In that case $J_{\mathrm{r}}=L$ in modulus, and the integration is one-dimensional over the range of available values of $J_{\mathrm{r}}$ :

$\Gamma^{\prime}\left(\varepsilon_{\mathrm{tr}}, J \simeq 0\right)=\int_{0}^{J_{\mathrm{r}}^{\max }} \chi^{\prime}\left(\varepsilon_{\mathrm{r}}^{*}, J_{\mathrm{r}}\right) \mathrm{d} J_{\mathrm{r}}$.

In the previous equation $\varepsilon_{\mathrm{r}}^{*}=\varepsilon_{\mathrm{tr}}-\varepsilon^{\dagger}$ with $\varepsilon^{\dagger}\left(J_{\mathrm{r}}\right)$ the energy of the centrifugal barrier.

The function $\chi$ to be integrated has been calculated exactly by Chesnavich \& Bowers (1977) in a number of situations for products with various shapes. However, coronene is a highly oblate molecule, and expressions are only available if the other product has a high symmetry. Therefore we had to restrict our study to sizes for which the product cluster is roughly spherical. From our previous results on cluster structure (Rapacioli et al. 2005a), two sizes fulfill this criterion to a good approximation, namely $n=3\left(B \approx 3.8 \times 10^{-5} \mathrm{~cm}^{-1}\right)$ and $n=12$ $\left(B \approx 1.4 \times 10^{-6} \mathrm{~cm}^{-1}\right)$. Thus our investigation of unimolecular dissociation will focus on the two parent clusters containing 4 or 13 molecules.

As a third ingredient, the PST calculation also needs an expression for the dissociation potential between the products, in order to locate the centrifugal barrier and estimate its height $\varepsilon^{\dagger}$. At long range $r \rightarrow \infty$ the interaction between a cluster of PAH molecules and a single PAH is essentially of the dispersion form, which we correct at short distances using the expression $-C_{6} /\left(r-r_{0}\right)^{6}$. The values of $C_{6}$ and $r_{0}$ were obtained from a series of Monte Carlo simulations with the distance between the two products constrained to increasing values. We find $C_{6}=$ $-4.3617 \times 10^{6} \mathrm{~kJ} \AA^{6} / \mathrm{mol}\left(\right.$ resp. $\left.C_{6}=-1.6574 \times 10^{7} \mathrm{~kJ} \AA^{6} / \mathrm{mol}\right)$ and $r_{0}=2.27 \AA$ (resp. $r_{0}=3.73 \AA$ ) for $n=4$ (resp. $n=13$ ).

\subsubsection{Calibration}

The statistical theory described previously can be used to calculate kinetic energy release (KER) distributions, given by Eq. (3) after normalization over $\varepsilon_{\text {tr }}$. The KER has the advantage over the rate constant that it does not include the unknown factor $C_{0}$, hence it provides a good testing ground for the accuracy of PST. 


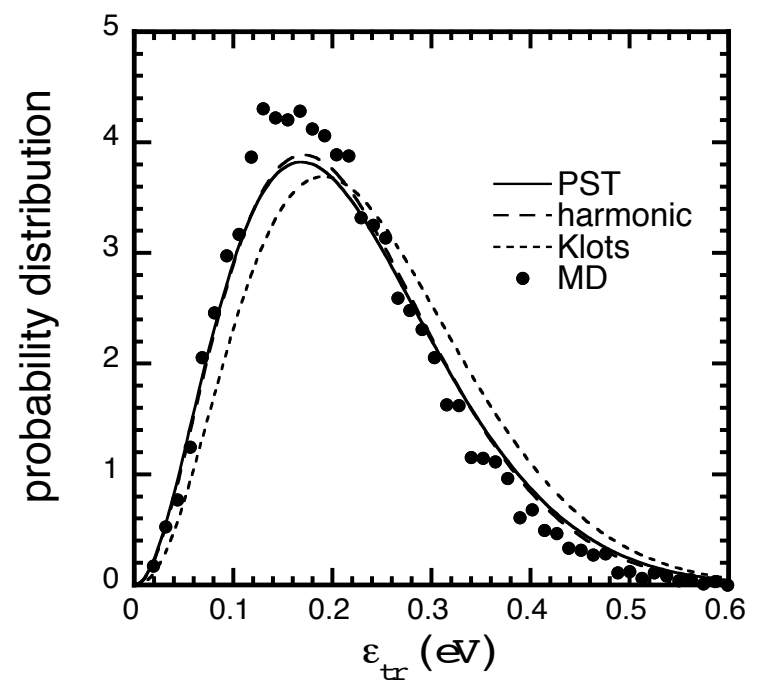

Fig. 5. Probability distribution of the kinetic energy released during the unimolecular evaporation of $\left(\mathrm{C}_{24} \mathrm{H}_{12}\right)_{4}$ at $2 \mathrm{eV}$ intermolecular energy obtained from MD simulations and from PST based on several approximations (see the text for details about these approximations).

For each of the two parent clusters, we performed a sample of 10000 molecular dynamics simulations at relatively high excess energy. For each trajectory, the cluster was initially prepared cold $(10 \mathrm{~K})$, and was suddenly heated. The simulation was ended when dissociation of a single molecule occured within 100 ps. Once evaporation was detected, the dissociation time was taken as the latest moment when the radial velocity of the dissociating molecule was pointing inward. Here the MD results can be considered as numerically exact, and as a reference for assessing the PST approach.

The distributions of KER obtained from the MD simulations are compared with PST predictions in Fig. 5 for the dissociation of $\left(\mathrm{C}_{24} \mathrm{H}_{12}\right)_{4}$ at $2 \mathrm{eV}$ excess energy.

The PST calculations were performed at three different levels of approximation. In its most rigorous form, the vibrational density of states is the anharmonic form extracted from Monte Carlo simulations, and the rotational density results from integrating Eq. (4) above. The harmonic approximation for the vibrational density is simply $\Omega(E) \propto E^{v-1}$ with $v=6 n-6$ the number of independent degrees of freedom in the system ( 3 rotational and 3 translational modes per molecule). The Klots approximation at low angular momentum (see Klots 1971; Calvo \& Parneix 2004, for more details) is similarly $\Gamma^{\prime}\left(\varepsilon_{\mathrm{tr}}\right) \propto \varepsilon_{\mathrm{tr}}^{\alpha}$ with $\alpha=(r-1) / 2$, $r=6$ being the number of rotational degrees of freedom in the products.

From Fig. 5 we generally find a very good agreement between the MD results and the PST predictions. In the case of the tetramer, the harmonic approximation for the vibrational DOS performs well. This is consistent with the existence of only a single (stacked) isomer for this cluster at the energies considered here. The Klots approximation for the rotational density does not affect the results quantitatively. This indicates that the results should be poorly sensitive to the exact shape of the products. For the larger cluster (distributions not plotted) the agreement remains very good but anharmonicities are found to be more significant.

The present agreement between molecular dynamics data and the PST results is encouraging and allows us to fit the missing parameter $C_{0}$ to reproduce the rate constant obtained from MD. Subsequently the statistical properties can be calculated from PST for any excitation energy. This is especially interesting at low energies, for which molecular dynamics simulations are not practical.

\subsubsection{Evaporation rates}

The variations of the rate constant with internal energy are represented in Fig. 7 for the two parent clusters. The physical contents of these results will be discussed later in the light of other competing decay channels.

An independent validation of phase space theory is obtained by comparing the rates from sets of molecular dynamics simulations performed at different energies to the PST predictions. We thus performed additional simulations for intermolecular energies in the 1.5-1.9 eV range for $\left(\mathrm{C}_{24} \mathrm{H}_{12}\right)_{4}$ and at $5.5 \mathrm{eV}$ for $\left(\mathrm{C}_{24} \mathrm{H}_{12}\right)_{13}$. The results shown in Fig. 7 are in very good agreement with the statistical theory, even though a logarithmic scale has been used for the graph. The discrepancy is found to be at most a factor 1.85 , which is satisfactory for a rate constant known to span orders of magnitude.

\section{Competition between evaporation and IR cooling}

Infrared emission is a possible relaxation energy channel for an excited cluster and can be in competition with evaporation. While evaporation mainly involves the intermolecular energy, most of the IR emission is driven by intramolecular modes. Therefore, in order to compare the evaporation and IR emission rates, the inter- and intramolecular energies must be first related to each other.

\subsection{Energy distribution}

In the next subsection, the spontaneous IR emission rates are calculated for each molecule against their intramolecular energy $E_{\text {intra }}$. The evaporation rates as a function of intermolecular energy $E_{\text {inter }}$ are given in Sect. 3.2.3. $E_{\text {intra }}$ and $E_{\text {inter }}$ both have to be expressed as a function of the total cluster energy $E_{\text {tot }}$. We first assume that the PAH cluster remains at thermal equilibrium. Because most intramolecular modes have a high frequency, the zero-point effects are important (Schmidt et al. 2006). The intermolecular modes, on the other hand, are all essentially soft and the intermolecular energy is equally distributed among those modes following classical equipartition.

We calculate $E_{\text {intra }}$ explicitly as a function of temperature from the known vibrational frequencies $\left\{\omega_{i}\right\}$ of the single coronene molecule:

$E_{\text {intra }}(T)=\sum_{i} \hbar \omega_{i}\left(\frac{1}{2}+\frac{1}{\exp \left(\hbar \omega_{i} / k_{\mathrm{B}} T\right)-1}\right)$,

$k_{\mathrm{B}}$ being the Boltzmann constant. The frequencies $\left\{\omega_{i}\right\}$ are obtained from the DFT calculations of Martin (1996) (which are more accurate than the present TB model).

Similarly, we calculate $E_{\text {inter }}$ as a function of temperature using the intermolecular frequencies from Rapacioli et al. (2005a). The total energy of the cluster is the sum of the intra- and intermolecular energies. Figure 6 represents the variations of the intramolecular energy per molecule $E_{\text {intra }}$ and of the total intermolecular energy $E_{\text {inter }}$ against $E_{\text {tot }}$. These curves were fitted using polynomial expressions of the form:

$\begin{aligned} E_{\mathrm{tot}} & =\sum a_{k}\left(E_{\text {intra }}\right)^{k} \\ & =\sum b_{k}\left(E_{\text {inter }}\right)^{k}\end{aligned}$

with fitting coefficients given in Table 1 . 

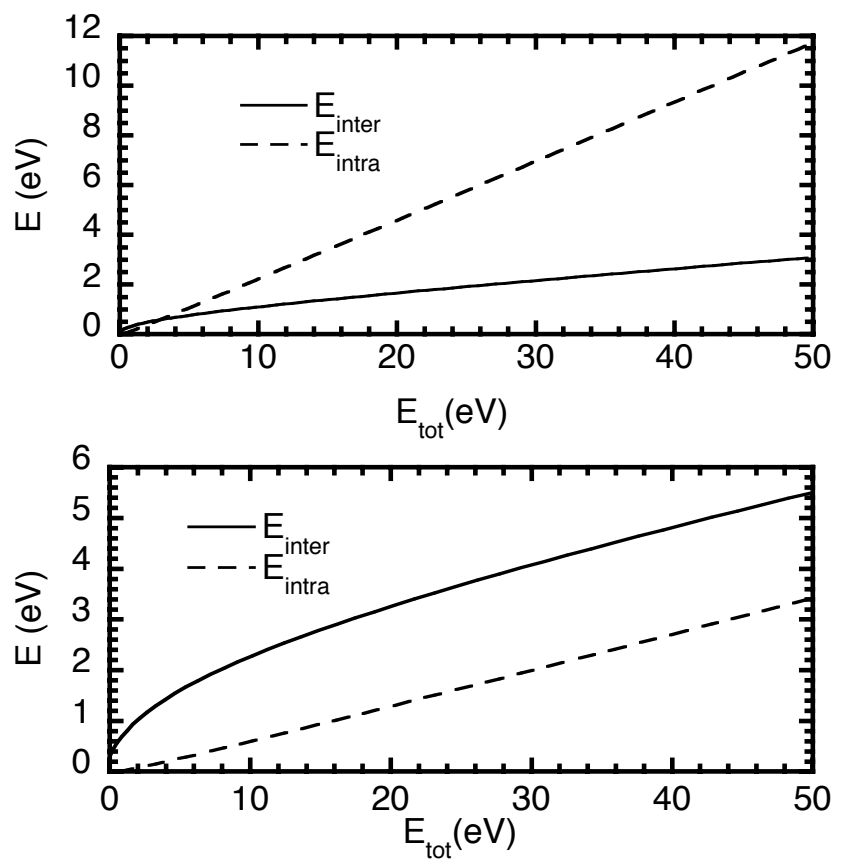

Fig. 6. Intramolecular energy per molecule and total intermolecular energy as a function of the total energy in the cluster for $\left(\mathrm{C}_{24} \mathrm{H}_{12}\right)_{4}$ (top panel) and $\left(\mathrm{C}_{24} \mathrm{H}_{12}\right)_{13}$ (bottom panel), after removal of harmonic zero-point energies.

\subsection{Spontaneous IR emission rates and evaporation rates}

We address here the estimation of IR emission rates for neutral coronene clusters. In a first approximation, the effects of clustering on the IR emission of a specific molecule in the cluster will be neglected. Thus the IR emission rate of the full cluster is obtained, considering the rates of individual molecules. The latter quantity explicitly depends on the energy $E_{\text {intra }}$. Monte Carlo type calculations of the IR emission of coronene molecules in interstellar conditions were reported by Joblin et al. (2002) (see also Mulas et al. 2006, for a more refined version of this approach). In this Monte Carlo approach, the IR emission rates are described with microcanonical statistics using an exact counting method for the density of states (Beyer \& Swinehart 1973) based on harmonic oscillators.

To discuss qualitatively the competition between the evaporation and IR emission processes, a first convenient step is to define a mean emission rate. This is achieved by summing the emission rates of all IR active vibrational modes of all molecules in the cluster:

$k_{\mathrm{IR}}\left(E_{\text {intra }}\right)=\sum_{n}^{N_{\mathrm{mol}}} \sum_{i, j} k_{\mathrm{IR}}^{n, v_{i} \rightarrow v_{i}-1}\left(v_{j}\right)\left(E_{\text {intra }}\right)$,

where $k_{\mathrm{IR}}^{v_{i} \rightarrow v_{i}-1}\left(v_{j}\right)$ is the spontaneous emission rate for a $v_{j}$ IR mode in the $v_{i} \rightarrow v_{i}-1$ transition at the available energy $E_{\text {intra }}$.

The variations of the mean IR emission rates $k_{\mathrm{IR}}$ and the evaporation rates $k_{\text {evap }}$ calculated in Sect. 3.2.3 for $\left(\mathrm{C}_{24} \mathrm{H}_{12}\right)_{4}$ and $\left(\mathrm{C}_{24} \mathrm{H}_{12}\right)_{13}$ are represented in Fig. 7 against $E_{\text {tot }}$. The clusters are assumed to be at thermal equilibrium, the energy repartition of Sect. 4.1 was used.

A critical energy $E^{*}$ can be defined as the energy of equal rates, $k_{\text {evap }}\left(E^{*}\right)=k_{\mathrm{IR}}\left(E^{*}\right)$. This energy is $10.4 \mathrm{eV}$ for $\left(\mathrm{C}_{24} \mathrm{H}_{12}\right)_{4}$ and $18.6 \mathrm{eV}$ for $\left(\mathrm{C}_{24} \mathrm{H}_{12}\right)_{13}$.
While the evaporation rates increase very strongly (exponentially) with $E_{\text {tot }}$, the IR emission rates show much smaller, polynomial-type variations. This suggests that the probability of evaporating a cluster before IR cooling strongly decreases for energies smaller than $E^{*}$ and becomes maximum for energies larger than this threshold.

\section{Modelling the photophysics of a neutral coronene cluster in a radiation field}

\subsection{Coronene cluster in a radiation field}

The Monte Carlo calculation of Joblin et al. (2002) is based on the exact stochastic method given by Gillespie (1978) and Barker (1983). The method consists of propagating "trajectories" that are Markovian random walks over the energy levels of the molecule. These trajectories depend on the following events: absorption of UV photons, emission of IR photons, but also fragmentation, as described in Joblin et al. (2006) . We have adapted this model to describe the photophysics of coronene clusters in a specific radiation field. The absorption rates were calculated using the absorption cross section $\sigma$ of the coronene cluster and a description of the astronomical radiation field. Since $\sigma$ is not known, we have scaled the cross section of the coronene molecule measured by Joblin et al. (1992) by the number of molecules. The IR emission rates as a function of $E_{\text {intra }}$ were described in Sect. 4.2. The evaporation rates were estimated by fitting the curves of Fig. 7 with $E_{\text {inter }}$ calculated from $E_{\text {tot }}$, as explained in Sect. 4.1.

The model allows us to calculate the time evolution of the internal energy and the associated events (photon absorption, emission, or fragmentation). Simulations are run over a given number of absorbed photons $\left(10^{6}\right.$ and $10^{7}$ for the lowest and highest absorption rates, respectively).

\subsection{Simulation of NGC 7023. The multiphoton absorption phenomenon}

The photophysical evolution of $\left(\mathrm{C}_{24} \mathrm{H}_{12}\right)_{4}$ and $\left(\mathrm{C}_{24} \mathrm{H}_{12}\right)_{13}$ clusters has been simulated in the environment of the northern photodissociation region (PDR) of NGC 7023. Based on the results of Rapacioli et al. (2005b), different regions corresponding to various depths inside the molecular cloud were selected. The depth is quantified by the selective extinction $A_{\mathrm{V}}$.

The radiation field in the different regions was calculated using the Meudon PDR code (Le Bourlot et al. 1993; Le Petit et al. 2006). The inputs for this calculation were modified with respect to previous calculations of Rapacioli et al. (2005b). For the total-to-selective extinction, we used a value for $R_{V}$ of 5.5 according to the recent study by Witt et al. (2006). The stellar spectrum used for HD 200775 is from the Kurucz library with a temperature of $15000 \mathrm{~K}$ (Kurucz 1991). The higher temperature derived by van den Ancker et al. (1997) was found to be inconsistent with the VUV flux observed on the star by the IUE satellite (see Valenti et al. 2000, and IUE archive at http://www.vilspa.esa.es/iue/iue.html). The radius of the star was taken as $10 R_{\odot}$ which is typical for a Be star. We performed the same analysis as in Rapacioli et al. (2005b) and inferred a hydrogen density of $7 \times 10^{3} \mathrm{~cm}^{-3}$ and a value $A_{\mathrm{V}}=0.41$ for the region of maximum emission in the $\mathrm{H}_{2} 9.7 \mu \mathrm{m}$ band.

The radiation field was calculated for $A_{\mathrm{V}}$ values of $0.41,1.0$, $1.6,2.5,3.5,5.0$ and 6.4 . The variations of the corresponding UV absorption rates are represented in Fig. 8. 

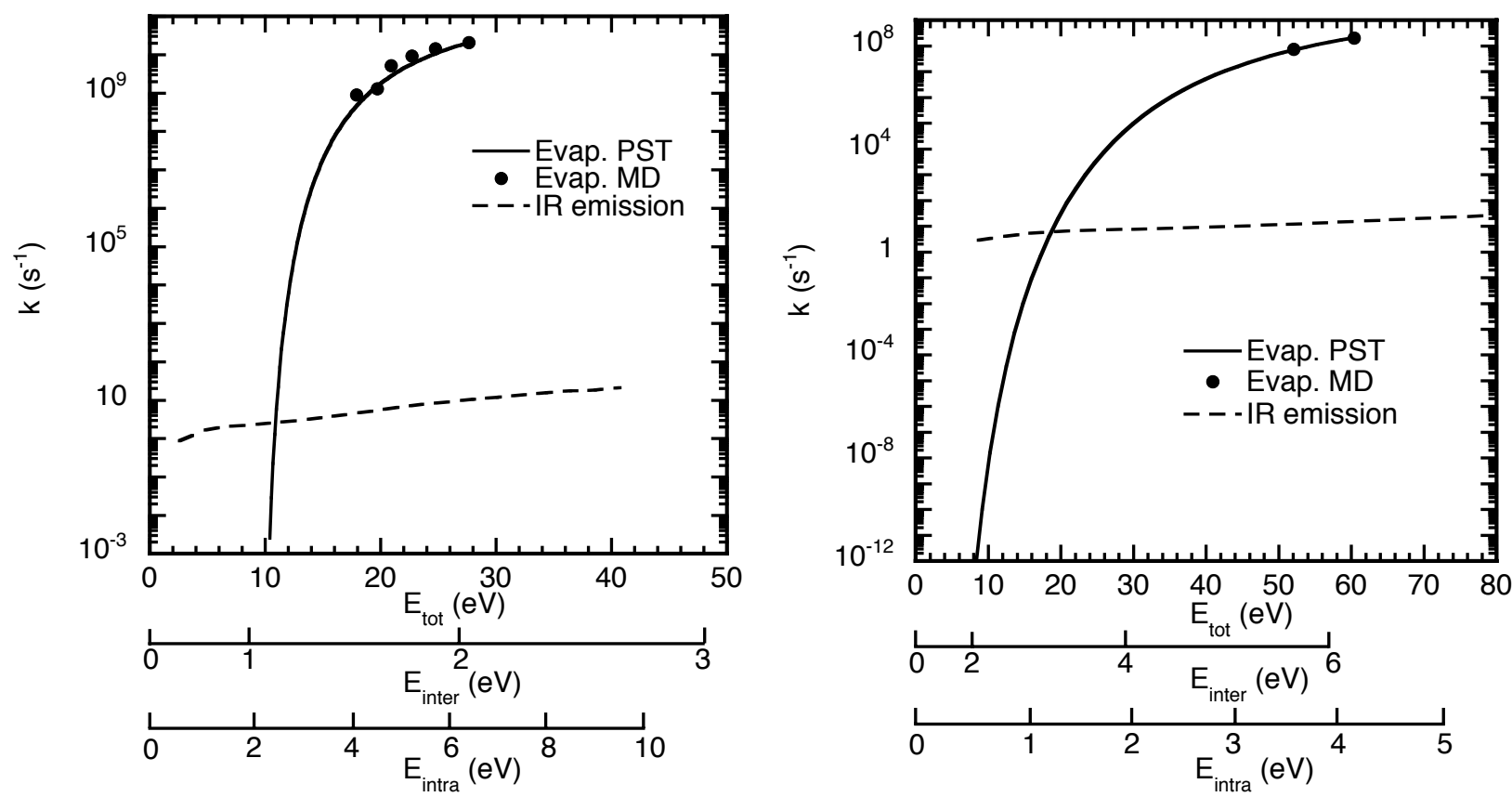

Fig. 7. Evaporation rates obtained with PST (solid lines) and MD simulations (symbols) as a function of the internal energy for coronene clusters with 4 (left panel) and 13 (right panel) molecules, respectively. The IR emission rates (Joblin et al. 2002) are also represented as dashed lines. The total, intra- and intermolecular energy scales are related to each other through Eqs. (6) and (7).

Table 1. Parameters of the polynomial fits of $E_{\text {tot }}$, as a function of $E_{\text {intra }}$ per molecule $\left(a_{k}\right)$ and $E_{\text {inter }}\left(b_{k}\right)$ for the (coronene) $)_{4}$ and (coronene) $)_{13}$ clusters.

\begin{tabular}{cccccc}
\hline \hline Cluster & $a_{0}$ & $a_{1}$ & $a_{2}$ & $a_{3}$ & $a_{4}$ \\
\hline$\left(\mathrm{C}_{24} \mathrm{H}_{12}\right)_{4}$ & 0.4345 & 4.2412 & 0 & 0 & 0 \\
$\left(\mathrm{C}_{24} \mathrm{H}_{12}\right)_{13}$ & 1.6614 & 13.9640 & 0 & 0 & 0 \\
\hline $\mathrm{Cluster}$ & $b_{0}$ & $b_{1}$ & $b_{2}$ & $b_{3}$ & $b_{4}$ \\
$\left(\mathrm{C}_{24} \mathrm{H}_{12}\right)_{4}$ & -0.0338 & -1.0037 & 12.3750 & -3.2847 & 3.498 \\
$\left(\mathrm{C}_{24} \mathrm{H}_{12}\right)_{13}$ & -0.2084 & -0.2720 & 2.4862 & -0.1666 & 0.00449 \\
\hline
\end{tabular}

The average timescales of evaporation are shown in Fig. 9 for $\left(\mathrm{C}_{24} \mathrm{H}_{12}\right)_{4}$ and $\left(\mathrm{C}_{24} \mathrm{H}_{12}\right)_{13}$. While the small evaporation timescale for $\left(\mathrm{C}_{24} \mathrm{H}_{12}\right)_{4}$ can be explained by the low critical energy $E^{*}$ of this cluster (see Sect. 4.2), the evaporation of the larger $\left(\mathrm{C}_{24} \mathrm{H}_{12}\right)_{13}$ has its critical energy above the cut-off of the radiation field. This is illustrated in Fig. 10, which displays the distributions of internal energy of the clusters prior to their evaporation. The mean energy for dissociation is in the range $10.5-11.5 \mathrm{eV}$ for $\left(\mathrm{C}_{24} \mathrm{H}_{12}\right)_{4}$ and $\sim 14.5 \mathrm{eV}$ for $\left(\mathrm{C}_{24} \mathrm{H}_{12}\right)_{13}$. This value is slightly shifted towards lower energies at $A_{\mathrm{V}}=5.0$ because of the extinction, which preferentially affects the more energetic photons. Another effect that can be observed in Fig. 10 is the absence of the high-energy tail for $A_{\mathrm{V}}=5.0$. Such events are only possible if the molecule can absorb two VUV photons without totally cooling by IR emission between the two absorptions, which requires in turn sufficiently high absorption rates.

From Fig. 9, it is clear that $\left(\mathrm{C}_{24} \mathrm{H}_{12}\right)_{13}$ clusters have accumulated the energy of more than one photon to reach the dissociation threshold, even in the more embedded regions. Figure 11 shows the internal energy contained in the clusters before absorption of the VUV photon leading to dissociation. In the case of the large clusters (13 units), a clear threshold is seen at $3 \mathrm{eV}$. This threshold is very sharp at low energies, as could be anticipated: there is no event in the $2.85 \mathrm{eV}$ channel of the histogram and $60-70 \%$ of the total number of events are found in the $3.0 \mathrm{eV}$

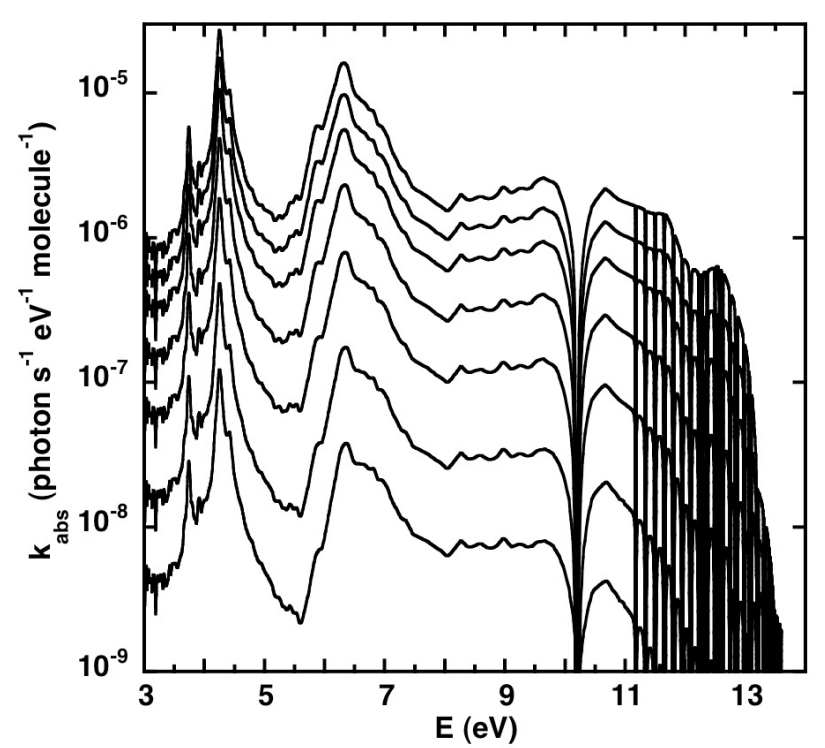

Fig. 8. Absorption rates of $\mathrm{C}_{24} \mathrm{H}_{12}$ at several depths inside the northern PDR of NGC 7023: $A_{\mathrm{V}}=0.41,1.0,1.6,2.5,3.5,5.0$ and 6.4 from top to bottom. For clusters, the absorption rates should be multiplied by the number of molecular units.

channel. This fraction drops very quickly at higher energies with $\sim 30 \%$ in the $3.25 \mathrm{eV}$ channel and the remaining $1-10 \%$ spread over channels up to $15.5 \mathrm{eV}$ and $13.5 \mathrm{eV}$ for $A_{\mathrm{V}}$ of 0.41 and 5.0, respectively.

The peak in the $3.0 \mathrm{eV}$ channel can be interpreted as the consequence of truncating the mean energy stored in the clusters at the threshold where the clusters are in equilibrium with the radiation field. Indeed, the model shows that a full dissipation of the energy absorbed by IR emission cannot be achieved before another photon is absorbed. At low internal energies, the 


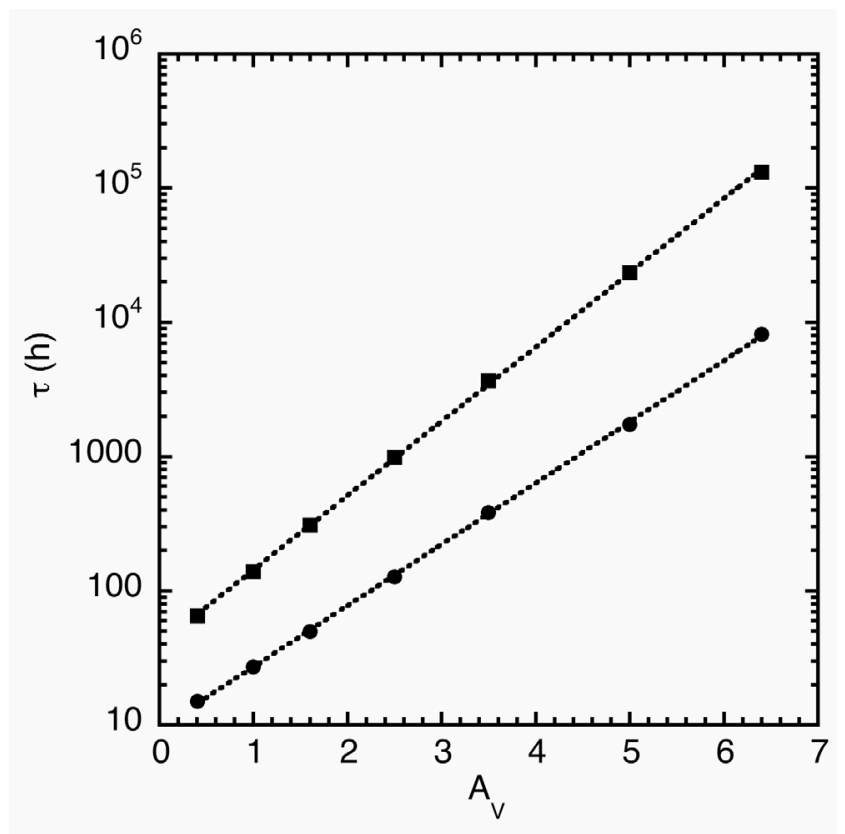

Fig. 9. Characteristic timescale for the evaporation of $\left(\mathrm{C}_{24} \mathrm{H}_{12}\right)_{4}$ (circles) and $\left(\mathrm{C}_{24} \mathrm{H}_{12}\right)_{13}$ (squares) as a function of $A_{\mathrm{V}}$ in the northern PDR of NGC 7023.

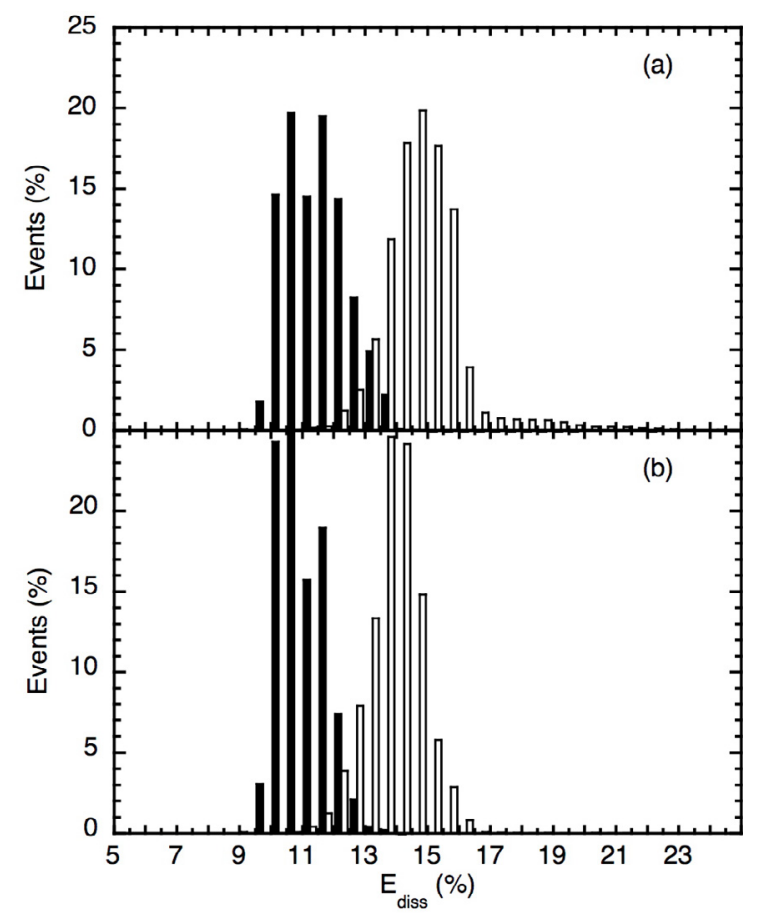

Fig. 10. Normalized distributions of the internal energy in $\left(\mathrm{C}_{24} \mathrm{H}_{12}\right)_{4}$ and $\left(\mathrm{C}_{24} \mathrm{H}_{12}\right)_{13}$ clusters prior to their evaporation (black and white bars respectively). The calculations were performed in two different radiation fields at a) $A_{\mathrm{V}}=0.41$ and b) $A_{\mathrm{V}}=5.0$.

cooling efficiency decreases as only low frequency modes with weak intensities are involved.

Contrary to large clusters, $\left(\mathrm{C}_{24} \mathrm{H}_{12}\right)_{4}$ species can be evaporated by a single VUV photon, multi-photon events playing only a minor role, as seen in Fig. 11. In this case, the $0 \mathrm{eV}$ channel corresponds to clusters that evaporate as soon as they absorb their first UV photon. The peak around $0.8 \mathrm{eV}$ corresponds to the mean energy stored in equilibrium with the radiation field.

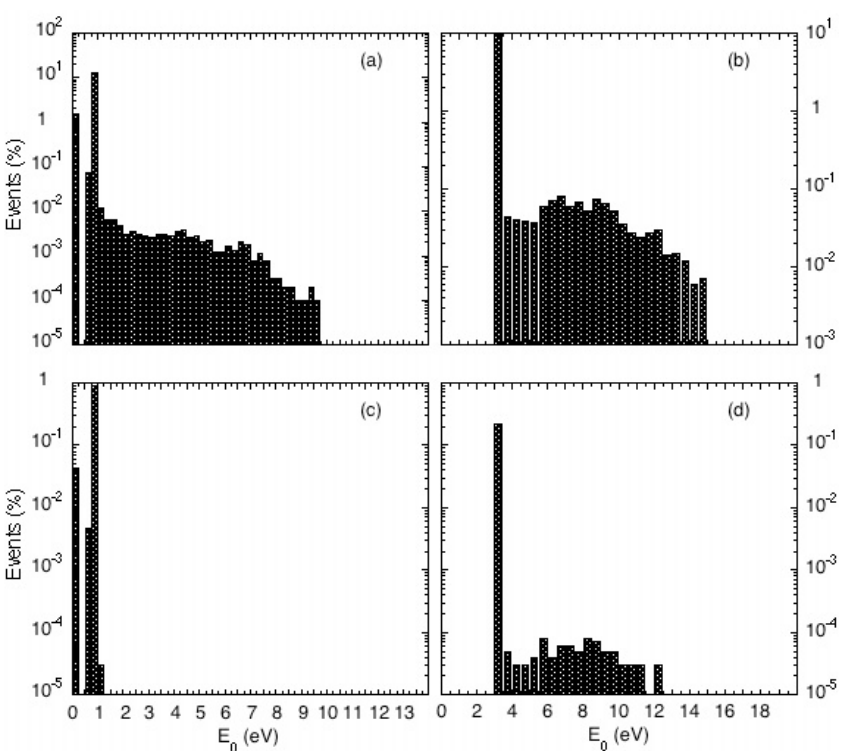

Fig. 11. Internal energy distributions of the clusters before absorption of the VUV photon leading to dissociation of $\left(\mathrm{C}_{24} \mathrm{H}_{12}\right)_{4}$ (left) and $\left(\mathrm{C}_{24} \mathrm{H}_{12}\right)_{13}$ (right). The calculations were performed in two different radiation fields at (a) and b)) $A_{\mathrm{V}}=0.41$ and (c) and d)) $A_{\mathrm{V}}=5.0$. The distributions are normalized to the total number of absorbed UV photons.

In this case, we can conclude from the observation of a population in the $0 \mathrm{eV}$ energy channel that accumulation of energy is not required for the evaporation of $\left(\mathrm{C}_{24} \mathrm{H}_{12}\right)_{4}$ clusters. This is obvious since energies lower than $13.6 \mathrm{eV}$ are already required.

\section{On the competition between formation and destruction of PAH clusters under interstellar conditions}

\subsection{Growth constants}

We estimate here the rate constant of the nucleation process associated with the growth of a single molecule on a cluster of $i-1$ molecules, both species being neutral. In the interstellar medium, the molecular gas can be considered as very rarefied, hence molecules and clusters have enough time to release their internal energy via IR emission before any collision occurs. In addition, we will assume that the molecular gas is thermalized, Boltzmann laws governing the distribution of translational and rotational energies of both clusters and single molecules.

If $\delta \tilde{n}_{i}$ represents the number of clusters of size $i$ formed upon the nucleation reaction $1+(i-1) \rightarrow i$ per unit of time $\delta t$ and inside a unit of volume, the growth constant $k_{i}$ is defined such that:

$\frac{\delta \tilde{n}_{i}}{\delta t}=k_{i} n_{1} n_{i-1}$.

In this equation $n_{1}$ and $n_{i-1}$ are the densities of single molecules and clusters of size $i-1$ per volume unit, respectively. $k_{i}$ can be expressed from standard collision theory (Landau et al. 1981) as:

$$
\begin{aligned}
k_{i}= & \iiint f_{1}\left(v_{1}\right) f_{i-1}\left(v_{i-1}\right)\left\|\boldsymbol{v}_{r}\right\| \sigma_{0} \\
& \times p_{i,+}\left(v_{r} ; E_{\mathrm{r}}\right) \mathrm{d}^{3} \boldsymbol{v}_{1} \mathrm{~d}^{3} \boldsymbol{v}_{i-1} f\left(E_{\mathrm{r}}\right) \mathrm{d} E_{\mathrm{r}} .
\end{aligned}
$$

In the above equation, $f_{1}\left(\boldsymbol{v}_{1}\right)$ and $f_{i-1}\left(\boldsymbol{v}_{i-1}\right)$ are the probability densities of the incident molecule and the cluster, which depend 
on their respective velocities $\boldsymbol{v}_{1}$ and $\boldsymbol{v}_{i-1}$ only. $f\left(E_{\mathrm{r}}\right)$ is the probability distribution of the rotational energy available at the beginning of the collision. $\sigma_{0}$ is the nucleation cross section, taken here as the geometrical cross section. $\boldsymbol{v}_{r}=\boldsymbol{v}_{1}-\boldsymbol{v}_{i-1}$ is the relative velocity vector. $p_{i,+}\left(v_{r} ; E_{\mathrm{r}}\right)$ is the probability that the collision leads to the formation of cluster size $i$. Assuming that $f_{1}\left(v_{1}\right)$ and $f_{i-1}\left(v_{i-1}\right)$ both follow a Boltzmann law at temperature $T$, Eq. (10) can be written more explicitly as:

$$
\begin{aligned}
k_{i}= & \left(\frac{\sqrt{m_{1} m_{i-1}}}{2 \pi k_{\mathrm{B}} T}\right)^{3} \iint\left\|\boldsymbol{v}_{r}\right\| p_{i,+}\left(\boldsymbol{v}_{r} ; E_{\mathrm{r}}\right) \sigma_{0} \\
& \times \exp \left[-\frac{\left(m_{1}+m_{i-1}\right) \boldsymbol{v}_{\mathrm{G}}^{2}+\mu \boldsymbol{v}_{r}^{2}}{2 k_{\mathrm{B}} T}\right] \mathrm{d}^{3} \boldsymbol{v}_{\mathrm{G}} \mathrm{d}^{3} \boldsymbol{v}_{\mathrm{r}} .
\end{aligned}
$$

In the latter equation, $m_{1}$ and $m_{i-1}$ are the masses of the incident molecule and the cluster, respectively; $v_{\mathrm{G}}$ is the velocity of the centre of mass of the entire system. The integration of this equation over $v_{\mathrm{G}}$ gives:

$$
k_{i}(T)=\frac{\sqrt{8 / \pi \mu}}{\left(k_{\mathrm{B}} T\right)^{3 / 2}} \iint E_{\mathrm{t}} \sigma_{0} p_{i,+}\left(E_{\mathrm{t}} ; E_{\mathrm{r}}\right) \mathrm{e}^{-E_{\mathrm{t}} / k_{\mathrm{B}} T} \mathrm{~d} E_{\mathrm{t}} f\left(E_{\mathrm{r}}\right) \mathrm{d} E_{\mathrm{r}},
$$

where $E_{\mathrm{t}}$ is the translational energy in the centre of mass reference frame, and $\mu$ the reduced mass of the system.

In order to calculate quantitative values of $k_{i}$, the collision cross section is approximated as the geometric area of the PAH molecule $\left(7.1 \times 10^{-19} \AA^{-2}\right)$. In Sect. 2.3 we only calculated values of $p_{i,+}\left(E_{\mathrm{t}} ; E_{\mathrm{r}}\right)$ for the specific case $E_{\mathrm{r}}=E_{\mathrm{t}}$. However a lower bound to $k_{i}$ can be estimated as follows.

At a given translational energy $E_{\mathrm{t}}$, the cluster growth probability decreases with the increase of rotational energy $E_{\mathrm{r}}$, as it implies an increase in the total energy. Hence

$p_{i,+}\left(E_{\mathrm{t}} ; E_{\mathrm{r}} \geq E_{\mathrm{r}}^{0}\right) \leq p_{i,+}\left(E_{\mathrm{t}} ; E_{\mathrm{r}}^{0}\right)$.

Similarly, at a given $E_{\mathrm{r}}$, the cluster growth probability decreases with increasing $E_{\mathrm{t}}$ :

$p_{i,+}\left(E_{\mathrm{t}} \geq E_{\mathrm{t}}^{0} ; E_{\mathrm{r}}\right) \leq p_{i,+}\left(E_{\mathrm{t}}^{0} ; E_{\mathrm{r}}\right)$.

These two trends have been checked using explicit atomic simulations, and performing a few tens of molecular dynamics trajectories of the nucleation process. A lower bound to $k_{i}$ can be calculated assuming that

$p_{i,+}\left(E_{\mathrm{t}} ; E_{\mathrm{r}}\right)= \begin{cases}p_{i,+}\left(E_{\mathrm{t}} ; E_{\mathrm{t}}\right) & \text { if } E_{\mathrm{t}} \geq E_{\mathrm{r}} \\ p_{i,+}\left(E_{\mathrm{r}} ; E_{\mathrm{r}}\right) & \text { if } E_{\mathrm{t}}<E_{\mathrm{r}} .\end{cases}$

Moreover, the collision constants $p_{i,+}$ provide an upper bound to the growth constants, by taking $p_{i,+}=1$ in Eq. (12).

Figure 12 shows the lower and upper limits to $k_{i}$ with $i=$ $2,3,4$ obtained in the temperature range $10^{2}-10^{4} \mathrm{~K}$. The two limits appear to be very close. This is due to the fact that, except for extremely high collisional energies, nearly all collisions lead to effective growth. In a first approximation, the growth rate can then be taken as the collision rate. We also note that the growth constant increases monotonically with temperature. Indeed a temperature increase enhances the rate of collisions which, for $T<2000 \mathrm{~K}$, nearly always lead to nucleation. Similar calculations have also been performed with the probability $p_{i,-}$ of destroying the parent cluster. The corresponding rates are found to be a few orders of magnitude smaller than the growth rates, hence we conclude that this process can be neglected.

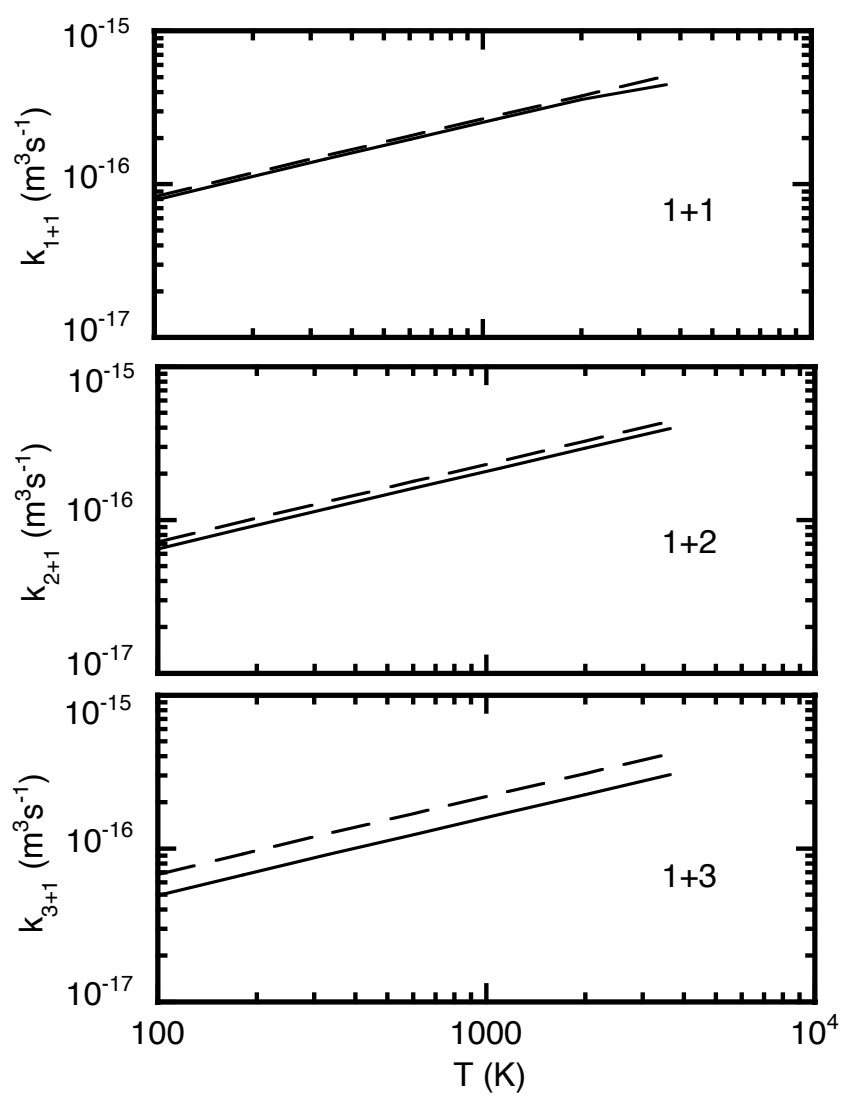

Fig. 12. Collision rates (dashed lines) and growth rates (solid lines) for the nucleation processes $1+1 \rightarrow 2,1+2 \rightarrow 3$ and $1+3 \rightarrow 4$ plotted as a function of temperature.

\subsection{Competition between formation and destruction in neutral PAH clusters}

In their study of the reflection nebula NGC 7023, Rapacioli et al. (2005b) gave hints that PAH molecules are likely to be condensed as clusters. These clusters are evaporated at the border of the cloud where isolated PAHs are observed. A minimum size of 400 carbon atoms for the observed clusters was also inferred from the observations.

An order of magnitude for the typical timescale of PAH clustering in this reflection nebula can be estimated from the calculations reported in Sect. 6 . We use a density $n_{\mathrm{H}}=7 \times 10^{3} \mathrm{~cm}^{-3}$ for NGC 7023, as discussed previously (Sect. 5.2), and a carbon abundance $n_{\mathrm{C}}=2 \times 10^{-4} n_{\mathrm{H}}$ following Sofia et al. (2004). We consider that about $20 \%$ of the carbon is contained in PAH molecules. For simplicity, and in order to apply our previous results, we also assume that all PAH molecules are coronene. This leads to a density of coronene molecules of $1.12 \times 10^{-2} \mathrm{~cm}^{-3}$. According to the PDR model, the kinetic temperature varies from $500 \mathrm{~K}$ to $60 \mathrm{~K}$ in the range $A_{\mathrm{V}}=0.41-6.4$.

We find that the aggregation timescale is typically $10^{4}-$ $8 \times 10^{4}$ years. This is much longer than the photoevaporation timescales derived for $\left(\mathrm{C}_{24} \mathrm{H}_{12}\right)_{4}$ and $\left(\mathrm{C}_{24} \mathrm{H}_{12}\right)_{13}$ in Sect. 5.2, which are below $1.5 \times 10^{5} \mathrm{~h}$ (17 years). Therefore, these clusters should be destroyed by the UV flux much faster than they can be reformed. This also implies that, when a large cluster starts to evaporate, the small fragments are also likely to photoevaporate themselves. It can be deduced from this theoretical work that, in the case of NGC 7023, the minimum size of the coronene clusters should be larger than 13 molecular units (312 carbon atoms), 
which is consistent with the minimum size of 400 carbon atoms inferred from the observations (Rapacioli et al. 2005b).

\subsection{Effects of ionization}

Until now we have focused on the description of neutral clusters. Our model can be used to constrain the size distribution of PAH clusters as a function of depth in the molecular cloud. One would like to be able to constrain the charge as well, similarly to what is done for single PAHs (Bakes et al. 2001; Li \& Draine 2002). While we can give hints about charge effects, fundamental studies are clearly required before considering further modeling.

Even though PAHs are likely to carry a charge in many objects of the ISM (Hudgins \& Allamandola 1999; Allamandola et al. 1989), Rapacioli et al. (2005b) have shown that the different populations of very small dust particles are spatially decorrelated in two PDRs NGC 7023 and $\rho$ Oph-SR3. As the distance from the stars increases, the mid-IR emission is found to be dominated first by positively ionized PAHs, then by neutral PAHs, and finally by VSGs. Similarly to single PAHs, PAH clusters could carry a charge. We expect this charge to be most likely positive, since the lifetime of anions will be limited by the easy photodetachment in these UV-irradiated regions where PAH clusters can be observed by their mid-IR emission. Charge effects can affect both the formation rate and the evaporation rate for different but related reasons.

Let us first consider the case of multiply charged systems. The nucleation between two charged subsystems seems very unlikely due to the strong Coulomb repulsion. We thus exclude the reactions of positively charged clusters with PAH cations. The stability of an existing assembly of PAH molecules will critically depend on its charge state, in addition to its size. Other factors, including the size of the PAH itself and the excitation mechanisms, presumably play an important role as well. In a multiply ionized cluster, Coulomb fragmentation will generally enhance the possibility of emitting single (charged) molecules. In addition, new fragmentation channels will be open if a single PAH molecule carries more than one charge, and various intermolecular and/or intramolecular Coulomb multifragmentation processes could take place. While the photofragmentation dynamics of a PAH assembly (or even a single PAH cluster) would be worth investigating in its own, it is beyond the scope of the present paper.

Based on previous theoretical calculations on small singly charged clusters of aromatic molecules such as benzene, naphtalene or anthracene (Piuzzi et al. 2002; Bouvier et al. 2002), cationic clusters are expected to be significantly more stable than their neutral counterparts from the energetic point of view. This increased stability reflects the charge delocalization over several molecules in the cluster, a collective effect which cannot be described using simple explicit potentials. Because the evaporation rate constant varies approximately exponentially with the dissociation energy, cationic clusters will be significantly more resistant to the decay of a neutral molecule.

In the region where PAH clusters are observed, PAHs are expected to be mainly neutral (Rapacioli et al. 2005b), and we can limit our discussion to the case where a neutral molecule collides with a $(\mathrm{PAH})_{n}^{+}$cluster. For the nucleation rate, the PAH molecule being nonpolar, the long-range ion/neutral interaction scales as $-C_{4} / r^{4}$ where the constant $C_{4}$ is proportional to the electronic dipole polarizability $\alpha$ of the neutral PAH. In a very simple approach, we use a Langevin model to estimate the collision rate constant. Based on our previous results on neutral/neutral collisions, we can reasonably assume that all collisions between a neutral PAH and a cationic cluster lead to an effective growth of the cluster. The Langevin prediction for the nucleation rate is then given by $k_{\text {nucl }}=2 \pi e q(\alpha / \mu)^{1 / 2}$ (see for instance Herbst 2001), where $e$ is the electronic charge, $\mathrm{q}$ the ionization degree ( $q=1$ for singly-charged clusters) and $\mu$ the reduced mass of the reactants. An average polarizability of the coronene molecule, as estimated from the components of the polarizability tensor via density-functional theory calculations (Malloci 2006), is $\alpha=46 \times 10^{-24} \mathrm{~cm}^{3}$. Using this value the nucleation rate is approximately $k_{\text {nucl }}=9 \times 10^{-10} / q \mathrm{~cm}^{3} \mathrm{~s}^{-1}$. Together with the PAH density derived previously for NGC 7023, this leads to aggregation timescales of $(2.0-3.5) / q 10^{3}$ years.

For the specific case of NGC 7023, considered in Sect. 6.2, the conclusion that small clusters are destroyed faster than they can be reformed could thus be modified if the clusters carry one single positive charge, as this would decrease their evaporation rate (mostly through increasing their binding energy) but also enhance the nucleation rates.

\subsection{Astrophysical implications}

As seen above, a single positive ionization is expected to stabilize PAH clusters with respect to evaporation, and to favor their formation by nucleation of a neutral molecule. Our conclusion that neutral PAH clusters are evaporated efficiently in the northern PDR of NGC 7023 without being reformed could thus be revised in the case of cationic clusters.

The linear scaling of the growth constant with inverse density suggests another mechanism that could affect the conditions of reformation. The northern PDR of NGC 7023 is heterogeneous, with some high-density filaments $\left(n \sim 10^{5}-10^{6} \mathrm{~cm}^{-3}\right)$ embedded in a more diffuse medium with $n \sim 10^{4} \mathrm{~cm}^{-3}$ (Fuente et al. 2000, and references therein). With a value of $5 \times 10^{5} \mathrm{~cm}^{-3}$ for the density, the timescale for formation could be lowered to $140-700$ years for a neutral cluster, and to 30-50 years for an ionized cluster. Considering the dissociation rates reported in Fig. 9 for neutral species, and taking into account the extra stability of the cationic species, the PAH clusters could thus survive much longer and closer to the border of the cloud. A scenario in which the growth of PAH clusters occurs in the PDR itself is however unlikely because it would involve the survival of dimers in PAH-emitting regions. This suggests that such clusters would be formed in the UV-shielded parts of the cloud. There is time to form them during the cycle of matter from diffuse to dense clouds. Indeed the quiet phase of molecular clouds will typically last $10^{6}$ years, allowing clusters of tens of molecules to be formed. The formation of young stars in these clouds creates PDRs, which are fed in with clusters and rapidly submitted to photoevaporation. Dynamical effects in PDRs such as an advancing photodissociation region (Lemaire et al. 1999; Fuente et al. 1999, 2000) could then bring these clusters into the $\mathrm{UV}$-irradiated regions.

\section{Conclusion}

The formation and physico-chemical evolution of PAHs is a question of great interest in astrochemistry. Rapacioli et al. (2005b) presented strong evidence that PAH clusters are good candidates for small carbonaceous grains. These authors suggested that those grains are indeed PAH clusters, and a minimum size of 400 carbon atoms per cluster was inferred in NGC 7023. In the present paper, the competition between the formation and destruction processes of such PAH clusters in the neutral state 
was investigated theoretically under realistic astrophysical conditions. Coronene clusters have been considered here as representative prototypes of PAH clusters.

The growth of neutral PAH clusters by nucleation of individual molecules was simulated using a model combining an explicit atomic force field to describe the intermolecular interactions, along with a quantum tight-binding approach for the intramolecular interactions. It was found that, under interstellar conditions, most of the collisions lead to cluster growth. This was interpreted as due to the large number of intramolecular modes that contribute to absorbing the excess collision energy.

Evaporation was treated in the framework of phase space theory, supplemented with molecular dynamics simulations. The clusters $\left(\mathrm{C}_{24} \mathrm{H}_{12}\right)_{4}$ and $\left(\mathrm{C}_{24} \mathrm{H}_{12}\right)_{13}$ were investigated, and a good agreement between the simulation results and the predictions of the statistical theory was found for the distribution of kinetic energy released after evaporation. The calculated evaporation rates were then introduced in an infrared emission model based on microcanonical statistics. This model allowed the simulation of these clusters in a specified interstellar radiation field.

When applied to the reflection nebula NGC 7023, our model indicates that $\left(\mathrm{C}_{24} \mathrm{H}_{12}\right)_{4}$ and $\left(\mathrm{C}_{24} \mathrm{H}_{12}\right)_{13}$ are both photoevaporated much faster than they can be reformed. In the case of $\left(\mathrm{C}_{24} \mathrm{H}_{12}\right)_{13}$, this evaporation is possible if the UV absorption rate is faster than the IR emission rate, so that at least $\sim 3 \mathrm{eV}$ of internal energy are stored in the cluster before absorption of a VUV photon. These results provide a plausible explanation for the absence of small VSGs observed in this PDR. They also suggest that PAH clusters are formed inside the molecular cloud and transported in the PDR by dynamical effects.

The present investigation is a first step towards the treatment of the complex dynamics of nucleation, thermal and photo fragmentations of PAH clusters in the interstellar medium. Our work could be extended by limiting the approximations done in the calculations of aggregation rates (impact parameter and initial energy distribution) and of evaporation rates (mainly the angular momentum). A next step could be to study larger clusters, especially those beyond the 400 carbon atom limit. It would be important to determine whether such large clusters could survive inside a reflection nebula like NGC 7023. Unfortunately, the computational efforts for simulating the evaporation of large clusters by molecular dynamics, to calculate their vibrational densities of states with Monte Carlo simulations, or to apply the IR/evaporation model will all tend to become extremely costly, at least one order of magnitude higher than in the present study. Further approximations will then become unavoidable for a proper extension of the present work.

Two important factors, namely ionization and density heterogeneities, might enhance the stability of PAH clusters in PDRs. These factors have been qualitatively discussed here. Only singly-charged cationic clusters are expected to be more stable than neutrals. Conversely, multicharged species and anionic clusters should be less stable due to electrostatic repulsion and electronic photodetachment processes, respectively. For the formation problem, it would be very useful to build a complete model to describe singly charged cationic assemblies of PAH molecules.

To be chemically relevant, such a model would need to include two main effects, that are not important for neutral clusters. Firstly, the charge will lead to $1 / r^{4}$ polarization contributions, or even many-body implicit interactions between induced dipoles at higher order. Secondly, the model should account for charge delocalization, the missing electron being able to hop between neighbouring molecules. In homogeneous molecular systems, the charge is not restricted to be carried by a single PAH, but may extend over several units, typically between two and four (Piuzzi et al. 2002). This phenomenon is known as charge resonance. Obviously, polarization and charge delocalization effects are not unrelated, but their many-body and quantum characters make them significantly harder to describe than the pairwise repulsiondispersion-electrostatic interactions in the presently investigated neutral clusters.

Thus, for cationic PAH clusters a more complex atomic model is necessary to take into account electron tranfer. A model along those lines was developed by Piuzzi et al. (2002) and Bouvier et al. (2002) in order to investigate the structure of small clusters of aromatic molecules. The extension of this approach to large clusters and/or large PAH molecules will be computationally demanding, considering the extensive sampling required in the study of unimolecular evaporation. In the context of nucleation, the coupling between intermolecular and intramolecular modes will further burden the application of this model. With respect to neutral clusters, the theoretical modeling of cationic PAH clusters is therefore expected to be more involved. Efforts in this direction will be invaluable to improve our understanding of the physical and chemical evolution of photodissociation regions.

Acknowledgements. We thank J. Le Bourlot for help with the PDR code and M. Schmidt for useful discussions. We gratefully acknowledge the anonymous referee for constructive comments. This work was supported by the GDR 2758 "Agrégation, fragmentation et thermodynamique des systèmes complexes isolés." We greatly thank the financial support from the Alexander von Humboldt Foundation. We also thank IDRIS for a generous allocation of computer resources.

\section{References}

Allamandola, L. J., Tielens, A. G. G. M., \& Barker, J. R. 1985, ApJ, 290, L25 Allamandola, L. J., Tielens, A. G. G. M., \& Barker, J. R. 1989, ApJS, 71, 733

Bakes, E. L. O., Tielens, A. G. G. M., \& Bauschlicher, Jr., C. W. 2001, ApJ, 556, 501

Barker, J. R. 1983, Chem. Phys., 77, 301

Benharash, P., Gleason, M. J., \& Felker, P. M. 1999, J. Phys. Chem. A., 103, 1442

Bernard, J. P., Boulanger, F., \& Puget, J. L. 1993, A\&A, 277, 609

Beyer, T., \& Swinehart, D. F. 1973, A. C. M. Com., 16, 379

Boulanger, F., Falgarone, E., Puget, J. L., \& Helou, G. 1990, ApJ, 364, 136

Bouvier, B., Brenner, V., Millié, P., \& Soudan, J.-M. 2002, J. Phys. Chem. A, 106, 10326

Bréchignac, P., Schmidt, M., Masson, A., et al. 2005, A\&A, 442, 239

Calvo, F., \& Parneix, P. 2004, J. Chem. Phys., 120, 2780

Cesarsky, D., Lequeux, J., Ryter, C., \& Gérin, M. 2000, A\&A, 354, L87

Chesnavich, W. J., \& Bowers, M. 1977, J. Chem. Phys., 66, 6

Fuente, A., Martin-Pintado, J., Rodriguez-Fernandez, N. J., et al. 1999, ApJ, 518, L45

Fuente, A., Martin-Pintado, J., Rodriguez-Fernandez, N. J., Cernicharo, J., \& Gerin, M. 2000, A\&A, 354, 1053

Gillespie, D. T. 1978, J. Comput. Phys., 28, 395

Gonzales, C., \& Lim, E. 1999, J. Phys. Chem., 103, 1437

Grimme, S. 2004, J. Comput. Chem., 25, 1463

Herbst, E. 2001, Chem. Soc. Rev., 30, 168

Hudgins, D. M., \& Allamandola, L. J. 1999, ApJ, 516, L41

Jarrold, M. F. 1991, in Clusters of Atoms and Molecules I, ed. H. Haberland (Springer)

Joblin, C., Léger, A., \& Martin, P. 1992, ApJ, 393, L79

Joblin, C., Toublanc, D., Boissel, P., \& Tielens, A. 2002, Mol. Phys., 22, 3595

Joblin, C., Toublanc, D., Pech, C., et al. 2006, in preparation

Klots, C. 1971, J. Phys. Chem., 75, 10

Kurucz, R. L. 1991, BAAS, 23, 1047

Landau, L., Lifshitz, E., \& Pitaevskii, L. 1981, in Course of Theoretical Physics, Physical Kinetics, Vol. 10 (Butterworth-Heinemann)

Le Bourlot, J., Pineau Des Forets, G., Roueff, E., \& Flower, D. R. 1993, A\&A, 267, 233

Le Petit, F., Nehmé, C., Le Bourlot, J., \& Roueff, E. 2006, A\&A, in press 
Léger, A., \& Puget, J. L. 1984, A\&A, 137, L5

Lemaire, J. L., Field, D., Maillard, J. P., et al. 1999, A\&A, 349, 253

Li, A., \& Draine, B. T. 2002, ApJ, 572, 232

Malloci, G. 2006, Private communication

Martin, J. 1996, Chem. Phys. Lett., 259, 679

Marzec, A. 2000, Carbon, 38, 1863

Miller, J. H., Mallard, W. G., \& Smyth, K. C. 1984, J. Phys. Chem., 88, 4963

Mulas, G., Malloci, G., Joblin, C., \& Toublanc, D. 2006, A\&A, submitted

Neirotti, J., Calvo, F., Freeman, L., \& Doll, J. 2000, J. Chem. Phys., 112, 10340

Piuzzi, F., Dimicoli, I., Mons, M., et al. 2002, Chem. Phys, 275, 123

Rapacioli, M., Calvo, F., Spiegelman, F., Joblin, C., \& Wales, D. J. 2005a, J. Phys. Chem. A., 109, 2487

Rapacioli, M., Joblin, C., \& Boissel, P. 2005b, A\&A, 429, 193
Schmidt, M., Masson, A., \& Bréchignac, C. 2006, Int. J. Mass Spec., 252, 173 Sofia, U. J., Lauroesch, J. T., Meyer, D. M., \& Cartledge, S. I. B. 2004, ApJ, 605, 272

Song, J. K., Lee, N. K., Kim, J. H., Han, S. Y., \& Kim, S. K. 2003, J. Chem. Phys., 119, 3071

Valenti, J. A., Johns-Krull, C. M., \& Linsky, J. L. 2000, ApJS, 129, 399

van de Waal, B. 1983, J. Chem. Phys., 79, 3948

van den Ancker, M. E., The, P. S., Tjin A Djie, H. R. E., et al. 1997, A\&A, 324, L33

Van Oanh, N. T., Parneix, P., \& Bréchignac, P. 2002, J. Phys. Chem. A., 106, 10144

Weerasinghe, S., \& Amar, F. G. 1993, J. Chem. Phys., 98, 4967

Witt, A. N., Gordon, K. D., Vijh, U. P., et al. 2006, ApJ, 636, 303 